
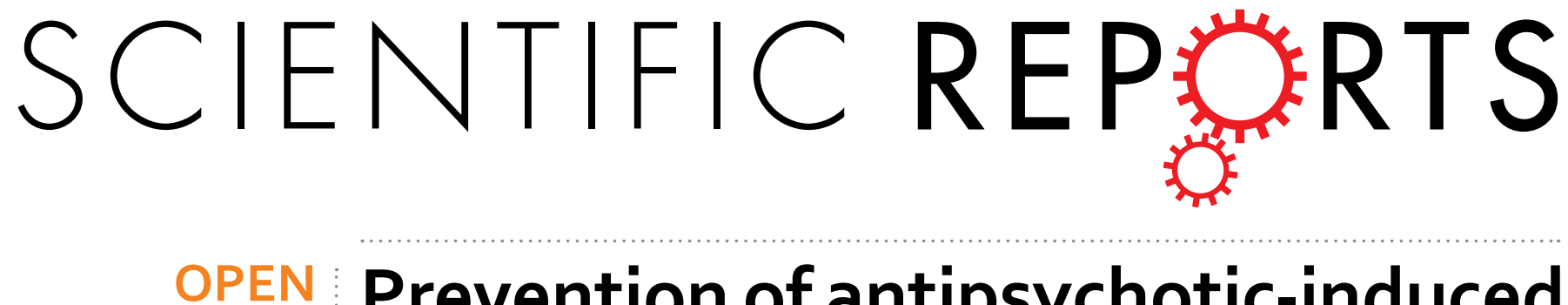

\title{
Prevention of antipsychotic-induced hyperglycaemia by vitamin D: a data mining prediction followed by experimental exploration of the molecular mechanism
}

Received: 08 January 2016

Accepted: 29 April 2016

Published: 20 May 2016

\author{
Takuya Nagashima ${ }^{1}$, Hisashi Shirakawa ${ }^{1}$, Takayuki Nakagawa ${ }^{2} \&$ Shuji Kaneko ${ }^{1}$
}

Atypical antipsychotics are associated with an increased risk of hyperglycaemia, thus limiting their clinical use. This study focused on finding the molecular mechanism underlying antipsychotic-induced hyperglycaemia. First, we searched for drug combinations in the FDA Adverse Event Reporting System (FAERS) database wherein a coexisting drug reduced the hyperglycaemia risk of atypical antipsychotics, and found that a combination with vitamin $D$ analogues significantly decreased the occurrence of quetiapine-induced adverse events relating diabetes mellitus in FAERS. Experimental validation using mice revealed that quetiapine acutely caused insulin resistance, which was mitigated by dietary supplementation with cholecalciferol. Further database analysis of the relevant signalling pathway and gene expression predicted quetiapine-induced downregulation of Pik3r1, a critical gene acting downstream of insulin receptor. Focusing on the phosphatidylinositol 3-kinase (PI3K) signalling pathway, we found that the reduced expression of Pik3r1 mRNA was reversed by cholecalciferol supplementation in skeletal muscle, and that insulin-stimulated glucose uptake into $\mathrm{C} 2 \mathrm{C} 12$ myotube was inhibited in the presence of quetiapine, which was reversed by concomitant calcitriol in a PI3Kdependent manner. Taken together, these results suggest that vitamin $D$ coadministration prevents antipsychotic-induced hyperglycaemia and insulin resistance by upregulation of PI3K function.

Atypical antipsychotics such as quetiapine and olanzapine are clinically used to treat a wide variety of mental disorders, including schizophrenia, bipolar disorder, depression and sleep disorders. However, these drugs are often associated with the occurrence of hyperglycaemia and other adverse events, thus limiting their clinical use $^{1,2}$. Antipsychotic-induced hyperglycaemia frequently leads to new-onset diabetes mellitus (DM), occasionally results in life-threatening diabetic ketoacidosis and coma, and, in the worst-case scenario, culminates in death ${ }^{3}$.

Despite wide recognition of the relationship between atypical antipsychotics and hyperglycaemia/DM, the pathogenesis of drug-induced DM remains poorly understood. Proposed mechanisms for antipsychotic-induced DM include weight gain, decreased insulin secretion from pancreatic $\beta$-cells, insulin resistance and impaired leptin action ${ }^{4}$. Although some clinical studies show that the antidiabetic agent, metformin, is effective against antipsychotic-associated DM, additional treatments are required because antipsychotic-induced hyperglycaemia is multifactorial, which makes it difficult to treat with a single-drug therapy ${ }^{5}$.

The FDA Adverse Event Reporting System (FAERS) is the largest worldwide database of the self-reports of adverse drug events, and freely available to the public. Several researchers have successfully identified previously unknown drug-drug interactions through analysis of FAERS ${ }^{6-8}$. In particular, Zhao and colleagues developed a FAERS-based approach to identify a concomitant drug B that might mitigate the risk of adverse events associated with the use of a drug $A^{8}$. Here, we first explored the FAERS database to determine an effective drug combination to lower the occurrence of antipsychotic-induced hyperglycaemia, and then ascertained the validity of the

${ }^{1}$ Department of Molecular Pharmacology, Graduate School of Pharmaceutical Sciences, Kyoto University, 46-29 Yoshida-Shimoadachi-cho, Sakyo-ku, Kyoto 606-8501, Japan. ${ }^{2}$ Department of Clinical Pharmacology and Therapeutics, Kyoto University Hospital, 54 Shogoin-Kawahara-cho, Sakyo-ku, Kyoto 606-8507, Japan. Correspondence and requests for materials should be addressed to S.K. (email: skaneko@pharm.kyoto-u.ac.jp) 


\begin{tabular}{|l|c|c|c|}
\hline Drug A & DM without drug A (\%) & DM with drug A (\%) & Odds ratio (95\% CI) \\
\hline Quetiapine & $37373 / 4482388(0.83)$ & $10809 / 65453(16.51)$ & $23.53(22.99-24.07)$ \\
\hline Olanzapine & $41668 / 4515358(0.92)$ & $6514 / 32483(20.05)$ & $26.93(26.17-27.72)$ \\
\hline Risperidone & $44382 / 4513579(0.98)$ & $3800 / 34262(11.09)$ & $12.56(12.13-13.01)$ \\
\hline Aripiprazole & $46007 / 4518947(1.02)$ & $2175 / 28894(7.53)$ & $7.91(7.57-8.28)$ \\
\hline Ziprasidone & $46569 / 4534867(1.03)$ & $1613 / 12974(12.43)$ & $13.68(12.98-14.43)$ \\
\hline Clozapine & $47124 / 4517820(1.04)$ & $1058 / 30021(3.52)$ & $3.47(3.26-3.69)$ \\
\hline
\end{tabular}

Table 1. Association between atypical antipsychotics (drug A) and the occurrence of DM in FAERS. Representative atypical antipsychotics were compared. The search terms for "DM" (including related adverse events) are described in Supplementary Table S1.

prospective drug combination in a mouse model of antipsychotic-induced glucose intolerance. We also predicted the molecular mechanism of antipsychotic-induced hyperglycaemia by combining metabolic pathway data from the Kyoto Encyclopaedia of Genes and Genomes (KEGG) PATHWAY database ${ }^{9,10}$ with a comprehensive rat toxicogenomics database, DrugMatrix. The DrugMatrix database contains microarray gene expression profiles ${ }^{11}$ deposited in the Gene Expression Omnibus (GEO) of the United States National Centre for Biotechnology Information ${ }^{12,13}$. Experimentally, we performed a reverse transcription-polymerase chain reaction (RT-PCR) analysis to confirm the predicted changes in gene expression in murine skeletal muscle. We further validated the hypothesis by in vitro experiments using C2C12 mouse myoblast cell line. The results of the current data mining study with experimental validation suggest that vitamin D can prevent quetiapine-induced hyperglycaemia through inhibition of insulin resistance via the upregulation of phosphatidylinositol 3-kinase (PI3K) function.

\section{Results}

Atypical antipsychotics increase the risk of DM-related adverse events in the FAERS database. We first investigated the association between atypical antipsychotic usage and DM-related adverse events in the FAERS database (Table 1). The search terms for DM-related adverse events are described in Supplementary Table S1. A disproportionality analysis of the database revealed associations between antipsychotic use and the increased occurrence of DM-related events. A strong association (odds ratio (OR) $>20$ ) was observed for quetiapine and olanzapine, and an intermediate association $(5<\mathrm{OR}<20)$ was observed for risperidone, aripiprazole and ziprasidone. Meanwhile, the association for clozapine was low $(\mathrm{OR}<5)$. These findings confirm the hypothesis that the risk of atypical antipsychotic-induced hyperglycaemia is properly reflected in database search. In the following analysis, we focused on quetiapine due to the high OR and the large number of DM reports linked to quetiapine usage.

Quetiapine-induced DM is observed regardless of confounding variables. We next investigated the involvement of confounding variables in quetiapine-induced, DM-related adverse events (Table 2). The association between quetiapine use and the increased risk of DM was observed even when each major adverse event search term (e.g., "diabetes mellitus", "diabetic ketoacidosis", "diabetic coma") was entered separately into the analysis. When affected individuals were stratified by age, the OR was greatest in the 40-49-year-old group, followed by the $<40$-year-old group, 50-59-year-old group, 60-69-year-old group and $>70$-year-old group, consistently with a previous pharmacoepidemiological study ${ }^{2}$. When individuals were stratified by gender, the increased occurrence of DM was observed in both male and female patients, with almost equal ORs. After restricting the patient population to those exhibiting specific primary diseases (e.g., schizophrenia or bipolar disorder), the DM frequency was still highly significant. Likewise, the increased incidence of DM was observed independently of concomitant psycholeptic agents, such as haloperidol or lithium. These results suggest that quetiapine use is significantly associated with an increased occurrence of DM-related adverse events, regardless of age, gender, primary diseases or combinatorial drug therapy.

Vitamin D decreases the occurrence of atypical antipsychotic-induced, DM-related adverse events. We next searched for coexisting drugs with the capacity to decrease the risk of quetiapine-induced $\mathrm{DM}$, irrespective of their molecular mechanisms of action (Table 3). Consequently, pregabalin, lamotrigine and vitamin D analogues (see Supplementary Table S2 for search terms for vitamin D analogues) were associated with a decreased occurrence of quetiapine-induced, DM-related adverse events. The lowest OR was observed for vitamin $\mathrm{D}$. The mitigating effect of vitamin $\mathrm{D}$ against $\mathrm{DM}$ was also observed in combinatorial therapy with olanzapine $(\mathrm{OR}=0.21,95 \%$ confidence interval $(\mathrm{CI})=0.14-0.32)$. Vitamin $\mathrm{D}$ itself did not affect the frequency of DM-related adverse events $(\mathrm{OR}=1.01,95 \% \mathrm{CI}=0.94-1.07, p=0.86)$, implying that vitamin $\mathrm{D}$ may interact with atypical antipsychotics to lessen the risk of hyperglycaemia. Below, we focused on vitamin $\mathrm{D}$ rather than the other candidate drugs, because pregabalin $(\mathrm{OR}=1.33,95 \% \mathrm{CI}=1.25-1.41)$ or lamotrigine $(\mathrm{OR}=1.41$, $95 \% \mathrm{CI}=1.30-1.52)$ was in itself associated with a slightly increased risk of DM-related adverse events.

Cholecalciferol alleviates quetiapine-induced hyperglycaemia and hyperinsulinemia in mice. To verify the prediction that vitamin D mitigates the risk of quetiapine-induced hyperglycaemia, we assessed the effect of the agent against quetiapine-induced glucose intolerance in an animal model (Fig. 1). Male ICR mice were fed a control or vitamin D (cholecalciferol)-supplemented diet (1200 IU cholecalciferol/day) for 1 week, fasted for $16 \mathrm{~h}$ and subjected to an intraperitoneal glucose tolerance test. Mouse body weights were unaffected by cholecalciferol diet (Supplementary Fig. S1). Administration of quetiapine (10 mg/kg, i.p.) to mice receiving 


\begin{tabular}{|l|c|c|c|}
\hline Group & DM without quetiapine (\%) & DM with quetiapine (\%) & Odds ratio (95\% CI) \\
\hline Limited by an adverse event name \\
\hline Diabetes mellitus & $20092 / 4482388(0.45)$ & $7668 / 65453(11.72)$ & $29.47(28.67-30.30)$ \\
\hline Diabetic ketoacidosis & $2897 / 4482388(0.06)$ & $1258 / 65453(1.92)$ & $30.30(28.35-32.39)$ \\
\hline Diabetic coma & $885 / 4482388(0.02)$ & $876 / 65453(1.34)$ & $68.69(62.55-75.44)$ \\
\hline Stratified by age & \multicolumn{5}{|l|}{} \\
\hline$<40$ years & $3622 / 657664(0.55)$ & $2229 / 16317(13.66)$ & $28.57(27.03-30.20)$ \\
\hline $40-49$ years & $3982 / 391629(1.02)$ & $2813 / 11828(23.78)$ & $30.38(28.82-32.02)$ \\
\hline $50-59$ years & $5929 / 547124(1.08)$ & $1690 / 10574(15.98)$ & $17.36(16.39-18.40)$ \\
\hline $60-69$ years & $5170 / 536481(0.96)$ & $409 / 4932(8.29)$ & $9.29(8.37-10.32)$ \\
\hline$\geq 70$ years & $4100 / 616472(0.67)$ & $175 / 5067(3.45)$ & $5.34(4.58-6.23)$ \\
\hline Stratified by gender & $14129 / 1572620(0.90)$ & $4512 / 24882(18.13)$ & $24.43(23.56-25.33)$ \\
\hline Male & $18996 / 2537316(0.75)$ & $6010 / 37798(15.90)$ & $25.06(24.30-25.85)$ \\
\hline Female & $1060 / 27277(3.89)$ & $1398 / 4160(33.61)$ & $12.52(11.45-13.68)$ \\
\hline Stratified by primary disease & $2459 / 10676(23.03)$ & $10.46(9.56-11.45)$ \\
\hline Schizophrenia & $649 / 23332(2.78)$ & $1219 / 2712(44.95)$ & $32.04(27.96-36.71)$ \\
\hline Bipolar disorder & $308 / 12393(2.49)$ & $785 / 3771(20.82)$ & $9.56(8.38-10.91)$ \\
\hline Stratified by drug combination & $351 / 13116(2.68)$ & & \\
\hline Haloperidol &
\end{tabular}

Table 2. Potential confounding variables for the quetiapine-induced DM in FAERS.

\begin{tabular}{|l|c|c|c|}
\hline Drug B & DM without drug B (\%) & DM with drug B (\%) & Odds ratio (95\% CI) \\
\hline Pregabalin & $10625 / 64076(16.58)$ & $184 / 1377(13.36)$ & $0.78(0.66-0.91)$ \\
\hline Lamotrigine & $10387 / 61398(16.92)$ & $422 / 4055(10.41)$ & $0.57(0.51-0.63)$ \\
\hline Vitamin D & $10743 / 64282(16.71)$ & $66 / 1171(5.64)$ & $0.30(0.23-0.38)$ \\
\hline
\end{tabular}

Table 3. Decrease of the occurrence of quetiapine-induced DM by a coexisting drug (drug B) in FAERS. The patient population was restricted to those receiving quetiapine. Top 3 coexisting drugs associated with decreased occurrence of DM-related adverse events are shown. The search terms for "vitamin D" are described in Supplementary Table S2.

the control diet acutely led to significantly higher levels of glucose (two-way analysis of variance (ANOVA): $F_{1,130}=24.32, p<0.001$ ) and insulin (two-way ANOVA: $F_{1,42}=4.74, p<0.05$ ) in the blood. On the other hand, dietary cholecalciferol supplementation significantly prevented the quetiapine-induced increases in both blood glucose and insulin (two-way ANOVA: $F_{1,130}=6.44, p<0.05 ; F_{1,42}=4.81, p<0.05$, respectively). The cholecalciferol diet did not modify the anti-psychostimulant effect of quetiapine on methamphetamine-promoted hyperlocomotion (Supplementary Fig. S2). Because hyperinsulinemia is a main feature of the insulin-resistant state ${ }^{14}$, our findings suggest that vitamin D averts quetiapine-induced hyperglycaemia by defending against insulin resistance.

Database search for signalling pathways and gene expression changes potentially responsible for quetiapine-induced insulin resistance. To clarify the molecular mechanisms underlying quetiapine-induced hyperglycaemia, we investigated toxicogenomics microarray datasets published in GEO (Accession No. GSE59923) by focusing on the expression of 24 gene products included in the KEGG "insulin resistance" pathway (Entry No. map04931; Fig. 2). In GEO, the expression levels of ten insulin resistance-related genes were downregulated in rat liver after oral quetiapine administration $(500 \mathrm{mg} / \mathrm{kg}, 5$ days of exposure with daily dosing; Table 4), while only one gene was upregulated. The ten downregulated genes encode nine relevant proteins downstream of insulin receptors, glycogen synthesis and tumour necrosis factor receptors. Among them, the most prominent reduction in expression was observed for Pik3r1, which encodes a known risk factor for DM, phosphatidylinositol 3-kinase (PI3K) ${ }^{15,16}$. Therefore, we speculated that vitamin D lessens quetiapine-induced hyperglycaemia by inhibiting the downregulation of Pik3r1/PI3K and subsequent induction of insulin resistance.

Cholecalciferol prevents quetiapine-induced downregulation of Pik3r1 in vivo. We next confirmed the prediction that vitamin D can prevent quetiapine-induced insulin resistance in a mouse model by examining the ability of cholecalciferol diet to block drug-provoked changes in gene expression related to the insulin receptor signalling pathway (Fig. 3). Immediately after administration of the glucose tolerance test in mice, mRNA levels were measured via quantitative RT-PCR in skeletal muscle, the predominant site of insulin-mediated glucose uptake ${ }^{17}$. Although not statistically significant (Welch's $t$-test: $p=0.079$ ), downregulation of Pik3r1 mRNA was observed even at $150 \mathrm{~min}$ after treatment with quetiapine. However, the quetiapine-facilitated downregulation of Pik3r1 was significantly reversed by dietary cholecalciferol supplementation (Kruskal-Wallis test 

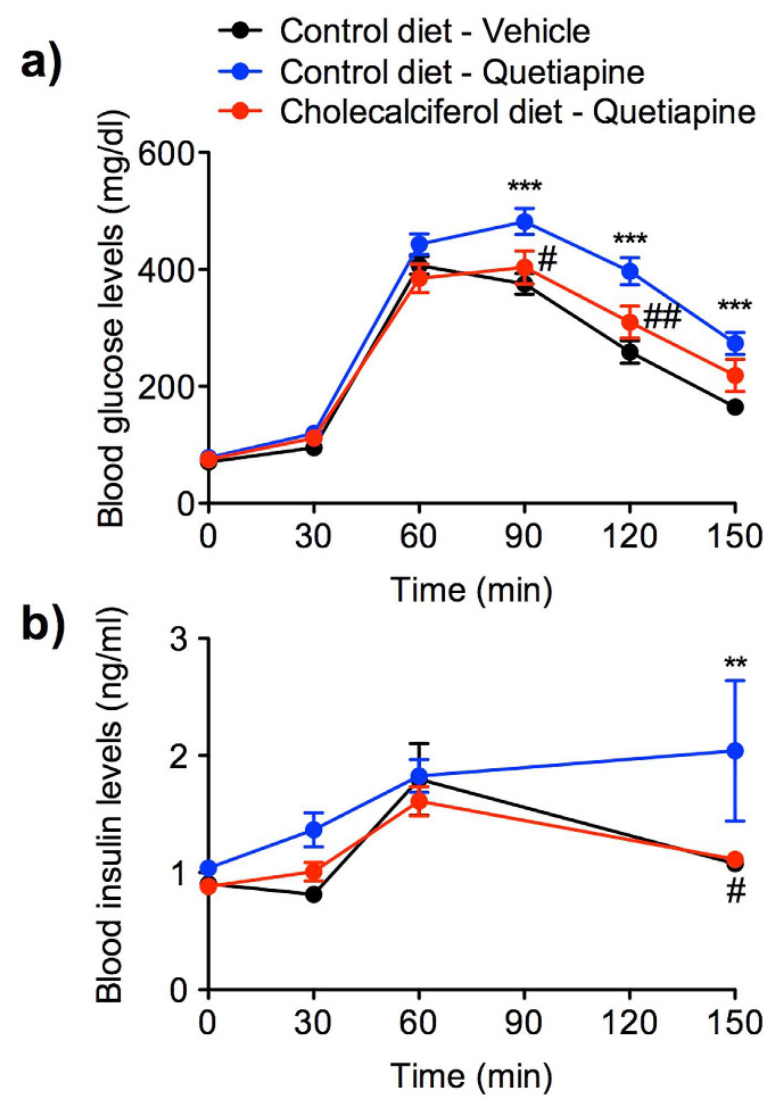

Figure 1. Effects of dietary vitamin $\mathrm{D} /$ cholecalciferol supplementation on quetiapine-induced increases in blood glucose and insulin levels. Mice were fed a control or cholecalciferol-supplemented diet (1200 IU cholecalciferol/day) for 1 week, fasted for $16 \mathrm{~h}$, and given quetiapine $(10 \mathrm{mg} / \mathrm{kg}$, i.p.) or vehicle $30 \mathrm{~min}$ before injection of D-glucose $(3 \mathrm{~g} / \mathrm{kg}$, i.p.). (a) Blood glucose $(n=14)$ and (b) blood insulin $(n=8)$ levels were measured at the indicated times. Data are given as means \pm standard error of the mean (SEM; ${ }^{* *} p<0.01$, ${ }^{* * *} p<0.001$ vs. vehicle-treated group; ${ }^{*} p<0.05,{ }^{\# \#} p<0.01$ vs. quetiapine-treated group).

value $=6.135, p<0.05$; Dunn's test, $p<0.05$ ). Significant upregulation of Insr (encoding the insulin receptor) was also observed in the cholecalciferol group (Kruskal-Wallis test value $=10.55, p<0.01$ ), consistently with a previous report ${ }^{18}$. No significant change was found in the expression levels of other relevant genes, including Irs 1 (encoding insulin receptor substrate 1 ; Kruskal-Wallis test value $=0.6154, p=0.7351$ ), Akt2 (encoding thymoma viral proto-oncogene 2; Kruskal-Wallis test value $=3.038, p=0.2189$ ) or Slc2a4 (encoding solute carrier family 2 (facilitated glucose transporter), member 4 ; Kruskal-Wallis test value $=1.885, p=0.3897$ ). These experimental data indicate that vitamin D counteracts quetiapine-induced insulin resistance by blocking Pik3r1 downregulation, validating the prediction obtained from analysis of the GEO and KEGG PATHWAY databases.

Calcitriol prevents quetiapine-induced insulin resistance via PI3K signalling pathway in vitro. We finally validated the hypothesis by glucose uptake assays using $\mathrm{C} 2 \mathrm{C} 12$ mouse myotubes (Fig. 4). Treatment with quetiapine $(1-100 \mu \mathrm{M})$ for $1 \mathrm{~h}$ inhibited the insulin-stimulated glucose uptake in a concentration-dependent manner. Significant inhibition was observed at 10 and $100 \mu \mathrm{M}$ quetiapine (Fig. 4a), suggesting the induction of insulin resistance. The insulin resistance induced by quetiapine $(100 \mu \mathrm{M})$ was improved by pretreatment with calcitriol (0.1-10 nM; 1,25-dihydroxycholecalciferol, the biologically active form of cholecalciferol/vitamin $\mathrm{D}_{3}$ ) for $24 \mathrm{~h}$ in a concentration-dependent manner, and significant improvement was achieved at $10 \mathrm{nM}$ calcitriol (Fig. 4b). Calcitriol pretreatment itself had no effect on the insulin-stimulated glucose uptake at any concentrations examined (Fig. 4c). The improvement of quetiapine-induced insulin resistance by calcitriol was still observed when co-treated with an AMP-activated protein kinase (AMPK) inhibitor compound C (20 $\mu \mathrm{M})$, but was significantly blocked in the presence of a PI3K inhibitor LY294002 (20 $\mu \mathrm{M}$, Fig. 4d). These data demonstrate that vitamin D improves quetiapine-induced insulin resistance via stimulating PI3K signalling pathway.

\section{Discussion}

The current data mining predictive analysis with experimental validation provides the first indication that vitamin D can prevent antipsychotic-induced hyperglycaemia by suppressing insulin resistance via upregulation of Pik3r1.

Little is known about the impact of vitamin D on metabolic abnormalities caused by atypical antipsychotics. Two pilot clinical studies assessed the efficacy of vitamin D against antipsychotic-induced metabolic side effects, 


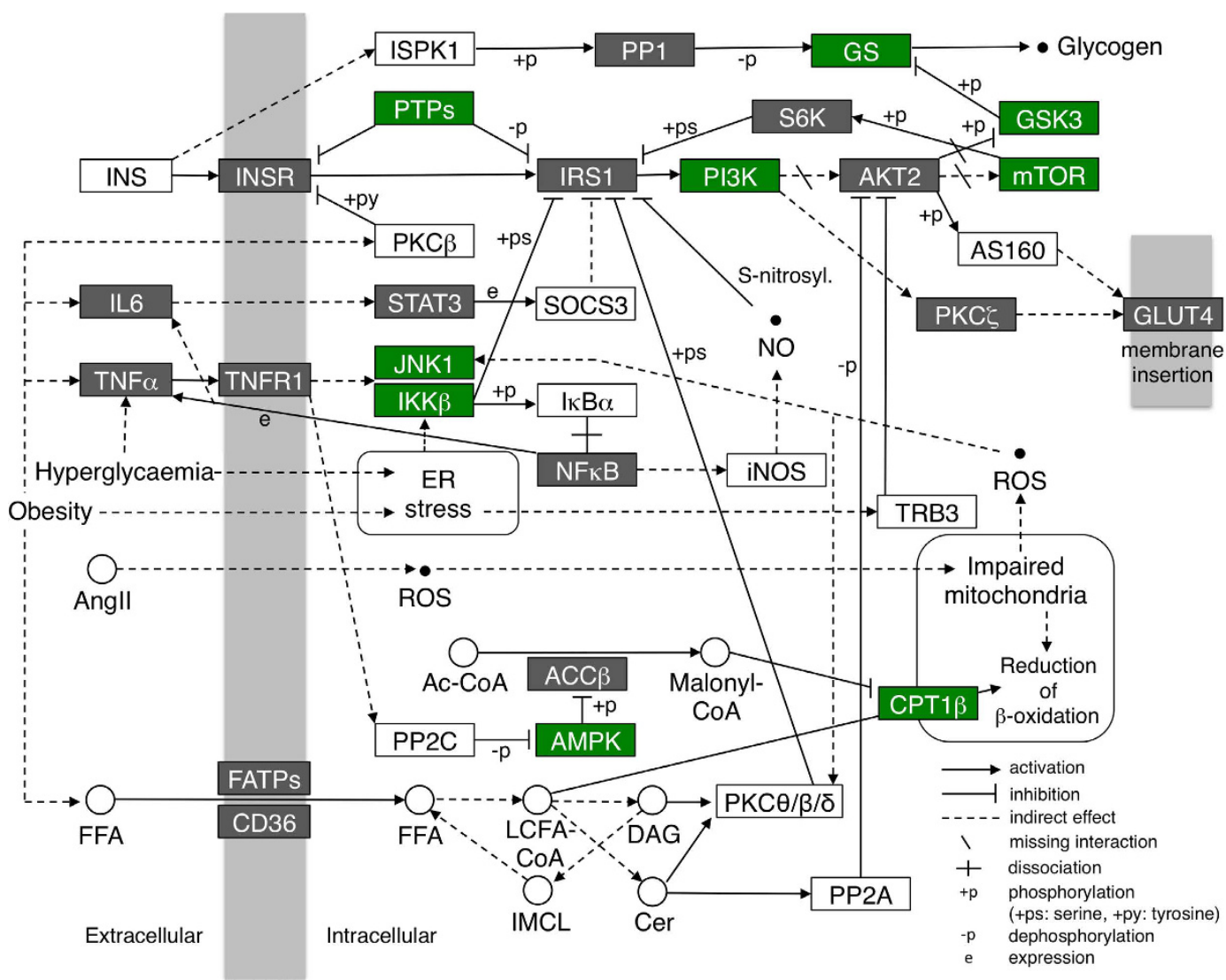

Figure 2. Candidate genes for action mechanism of quetiapine-induced insulin resistance, as predicted by combined analysis of GEO and KEGG PATHWAY databases. The insulin resistance pathway map in skeletal muscle cells was derived from the KEGG PATHWAY database (Entry No. map04931), with slight modifications. Changes in gene expression after oral quetiapine administration $(500 \mathrm{mg} / \mathrm{kg}, 5$ days of exposure with daily dosing) were investigated using GEO microarray data (GEO Accession No. GSE59923). Genes with expression levels measured in the microarray data are shown in green (significantly altered by quetiapine administration) or grey (not significantly altered by quetiapine administration). Ac-CoA = acetyl-coenzyme A. ACC $\beta=$ acetylcoenzyme A carboxylase $\beta$. AMPK $=$ AMP-activated protein kinase. AngII = angiotensin II. AS160 = Akt substrate of $160 \mathrm{kDa}$. Cer $=$ ceramide. $\mathrm{CPT} 1 \beta=$ carnitine palmitoyltransferase $1 \beta . \mathrm{DAG}=$ diacylglycerol. FATPs $=$ fatty acid transport proteins. FFA $=$ free fatty acid. GLUT4 = glucose transporter type $4 . \mathrm{GS}=$ glycogen synthase. GSK3 = glycogen synthase kinase 3 . IKK $\beta=$ inhibitor of nuclear factor-kappa-B kinase subunit $\beta$. IL6 = interleukin 6. IMCL = intramyocellular lipid. iNOS = inducible nitric oxide synthase. INS = insulin. $\mathrm{INSR}=$ insulin receptor. IRS $1=$ insulin receptor substrate $1 . \mathrm{ISPK} 1=$ insulin-stimulated protein kinase $1 . \mathrm{I} \kappa$ $\mathrm{B} \alpha=$ nuclear factor-kappa-B inhibitor $\alpha$. JNK1 = c-Jun N-terminal kinase 1. LCFA-CoA = long-chain fatty acyl-coenzyme A. $\mathrm{mTOR}=$ mechanistic target of rapamycin. $\mathrm{NF} \kappa \mathrm{B}=$ nuclear factor- $\kappa-\mathrm{B} . \mathrm{NO}=$ nitric oxide. $\mathrm{PI} 3 \mathrm{~K}=$ phosphatidylinositol 3-kinase. $\mathrm{PKC}=$ protein kinase $\mathrm{C} . \mathrm{PP}=$ protein phosphatase. $\mathrm{PTPs}=$ protein tyrosine phosphatases. $\mathrm{ROS}=$ reactive oxygen species. $\mathrm{S} 6 \mathrm{~K}=\mathrm{p} 70$ ribosomal $\mathrm{S} 6$ kinase. $\mathrm{SOCS} 3=$ suppressor of cytokine signalling 3 . STAT3 = signal transducer and activator of transcription 3 . TNFR1 = tumor necrosis factor receptor $1 . \mathrm{TNF} \alpha=$ tumor necrosis factor $\alpha . \mathrm{TRB} 3=$ tribbles homolog 3.

but both were limited by a statistically insufficient sample size ${ }^{19,20}$. Another investigation suggested the involvement of vitamin D deficiency in antipsychotic-induced hyperglycaemia ${ }^{21}$. However, this study drew its conclusion from animal models whose weight gain was suppressed by clozapine treatment, which is inconsistent with clinical observations. By contrast, our results are supported by the analysis of human clinical databases as well as pharmacological experiments both in vivo and in vitro, thus providing strong evidence and a molecular basis for the efficacy of vitamin D as a preventative treatment against antipsychotic-induced hyperglycaemia. Our findings are also in line with a previous report showing that vitamin D may prevent the development of type $2 \mathrm{DM}^{22}$.

The North American Association for the Study of Obesity reported an increased risk of DM for clozapine and olanzapine, an inconsistent risk for quetiapine and risperidone, and no risk for aripiprazole and ziprasidone ${ }^{1}$. Nevertheless, our FAERS analysis indicated that clozapine is associated with a relatively low risk of DM. This discrepancy might be explained by a lower number of adverse event reports for clozapine-induced DM in the FAERS database, possibly because glucose dysregulation following clozapine use is easily detected and rapidly managed via mandatory blood monitoring ${ }^{23}$. On the other hand, a relatively higher DM risk was found for aripiprazole and ziprasidone in FAERS, perhaps due to antipsychotic polypharmacy. In fact, our additional analysis of FAERS showed that nearly $85 \%$ (1368/1613) of DM patients who received ziprasidone also received quetiapine. 


\begin{tabular}{|l|l|c|c|c|}
\hline Probe name & Gene symbol (Gene title) & Encoding protein & Relative expression & $\boldsymbol{p}$-value \\
\hline NM_013005_PROBE1 & Pik3r1 (phosphatidylinositol 3-kinase, regulatory subunit, polypeptide 1) & PI3K & $0.39 \pm 0.13$ & 0.0143 \\
\hline NM_013200_PROBE1 & Cpt1b (carnitine palmitoyltransferase 1b, muscle) & CPT1 $\beta$ & $0.46 \pm 0.01$ & 0.0186 \\
\hline L27112_PROBE1 & Mapk9 (mitogen-activated protein kinase 9) & JNK1 & $0.46 \pm 0.08$ & 0.0021 \\
\hline X95577_PROBE1 & Prkab1 (protein kinase, AMP-activated, beta 1 non-catalytic subunit) & AMPK & $0.50 \pm 0.12$ & 0.0176 \\
\hline X73653_PROBE1 & Gsk3b (glycogen synthase kinase 3 beta) & GSK3 & $0.63 \pm 0.05$ & 0.0025 \\
\hline L37085_PROBE1 & Frap1 (FK506 binding protein 12-rapamycin associated protein 1) & mTOR & $0.66 \pm 0.03$ & 0.0001 \\
\hline NM_013089_PROBE1 & Gys2 (glycogen synthase 2) & GS & $0.67 \pm 0.06$ & 0.0050 \\
\hline AI172465_PROBE1 & Ptpn11 (protein tyrosine phosphatase, non-receptor type 11) & PTPs & $0.70 \pm 0.07$ & 0.0173 \\
\hline AF115282_PROBE1 & Ikbkb (inhibitor of kappaB kinase beta) & IKK $\beta$ & $0.70 \pm 0.08$ & 0.0396 \\
\hline Z29486_PROBE1 & Prkaa2 (protein kinase, AMP-activated, alpha 2 catalytic subunit) & AMPK & $0.74 \pm 0.05$ & 0.0113 \\
\hline A1598719_PROBE1 & Mapk8 (mitogen-activated protein kinase 8) & JNK1 & $1.30 \pm 0.09$ & 0.0489 \\
\hline
\end{tabular}

Table 4. GEO data showing the effects of quetiapine on the expression levels of insulin resistance-related genes. Expression levels are given as means \pm SEM relative to those of the vehicle (carboxymethylcellulose)treated group. $n=20-23$ for vehicle; $n=3$ for quetiapine-treated group.

The primary suspect drug for DM was quetiapine in $77.5 \%$ of these cases (1250/1613), as opposed to ziprasidone in only $5.6 \%$ of the cases $(91 / 1613)$.

Vitamin D mediates biological responses by binding to nuclear vitamin D receptors that function as transcription factors to regulate target gene expression ${ }^{24}$. Here, we found that dietary vitamin D supplementation upregulated Pik3rl in an animal model of antipsychotic-induced glucose intolerance. In addition, the improvement of antipsychotic-induced insulin resistance by calcitriol was blocked in the presence of PI3K inhibitor in our in vitro experiments, further supporting the functional involvement of Pik3r1. Mice lacking both Pik3r1 and Pik3r2 in the skeletal muscles exhibit severely impaired PI3K signalling and insulin resistance ${ }^{25}$. Akt is the major downstream effector of PI3K and promotes the membrane translocation of glucose transporter 4 (GLUT4), thus increasing cellular glucose uptake ${ }^{26}$. Altogether, these findings suggest that Pik3r1 downregulation promotes hyperglycaemia by impairing GLUT4-facilitated glucose uptake. Meanwhile, the upregulation of Ins $r$ by dietary vitamin D supplementation may help to overturn glucose intolerance.

Nonetheless, our data do not preclude other possible mechanisms of antipsychotic/vitamin D action. For example, atypical antipsychotics and vitamin D both reportedly affect insulin resistance by regulating the phosphorylation of insulin signalling pathway proteins (e.g., IRS1 and Akt) in a non-genomic manner ${ }^{27-29}$. Moreover, antipsychotics decrease insulin secretion from pancreatic $\beta$-cells by promoting cellular apoptosis ${ }^{30,31}$, while vitamin D improves pancreatic $\beta$-cell function ${ }^{22}$. Further investigation is required to determine to what extent the expression of $P i k 3 r 1$ and insulin resistance are affected by a long-term treatment with quetiapine and/or vitamin $\mathrm{D}$.

Databases like FAERS and GEO are useful tools for investigating drug toxicity, but data mining analyses are fraught with many limitations in terms of generating hypotheses. For instance, we could not obtain the preventive effect of quetiapine-induced DM for metformin by the analysis of FAERS (OR $=5.29,95 \% \mathrm{CI}=4.90-5.71$ ) because of the indication bias. In addition, even though a large number of toxicogenomics datasets are registered in GEO, the comprehensiveness of reported experimental conditions is presently insufficient. However, these shortcomings can be overcome by integration with systems pharmacology databases ${ }^{32}$ or by meta-analysis of large-scale toxicogenomic data ${ }^{33}$. Despite the challenges for prediction accuracy, data mining is still useful in areas where traditional clinical investigations and animal experiments fail to provide insights into molecular mechanisms of complicated conditions, such as antipsychotic-induced hyperglycaemia.

In conclusion, this study demonstrated the efficacy of vitamin D against antipsychotic-induced hyperglycaemia using data mining prediction followed by experimental validation both in vivo and in vitro. Based on the current results, we propose a novel vitamin D/antipsychotic combination pharmacotherapy in which vitamin $\mathrm{D}$ can efficaciously safeguard against antipsychotic-induced hyperglycaemia accompanied by insulin resistance. Thus, the analysis of rapidly growing databases of clinical adverse events and molecular toxicity can provide insights into the practical management of undesirable adverse drug events, as well as their molecular mechanisms.

\section{Materials and Methods}

Analysis of the FAERS database. FAERS adverse event reports were obtained from the FDA website (http://www.fda.gov/Drugs/GuidanceComplianceRegulatoryInformation/Surveillance/AdverseDrugEffects/). Duplicated reports (among a total of 5,821,354 reports) from the first quarter of 2004 through the second quarter of 2014 were filtered by applying the FDA's recommendation of adopting the most recent case number. Consequently, 4,547,841 remaining reports were analysed in this study. Arbitrary drug names, including trade names and abbreviations, were mapped into unified generic names via text mining. Adverse event risk was evaluated by calculating the reporting ORs with a 95\% CI according to methods described in the literature ${ }^{34}$.

Briefly, individuals in the FAERS database were divided into the following four groups: (a) individuals who received the drug of interest (i.e., quetiapine or vitamin D) and exhibited DM-related adverse events; (b) individuals who received the drug of interest, but did not exhibit DM-related adverse events; (c) individuals who did not receive the drug of interest and exhibited DM-related adverse events; and (d) individuals who did not receive the drug of interest and did not exhibit DM-related adverse events. The OR with 95\% CI was defined as follows: 
a)

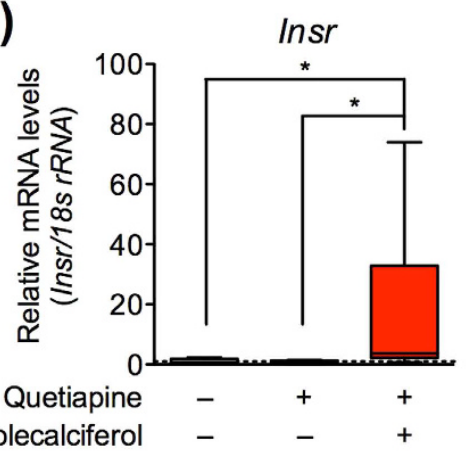

b)

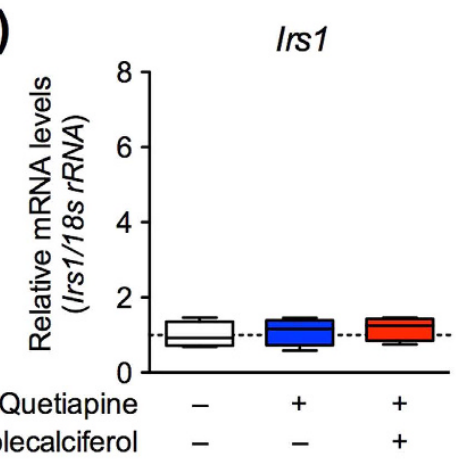

c)

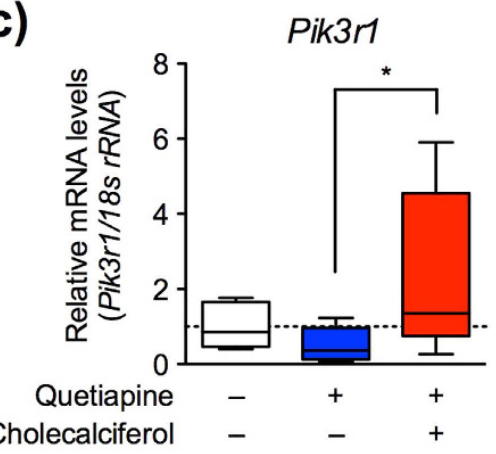

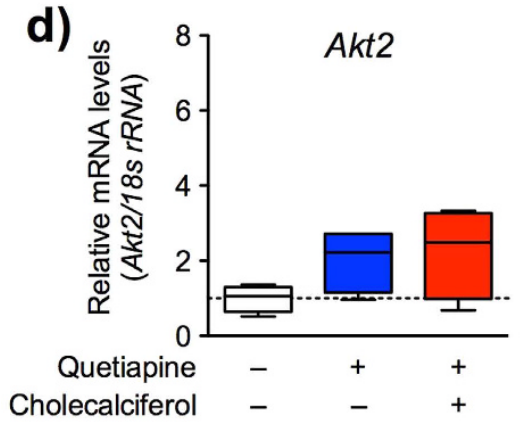

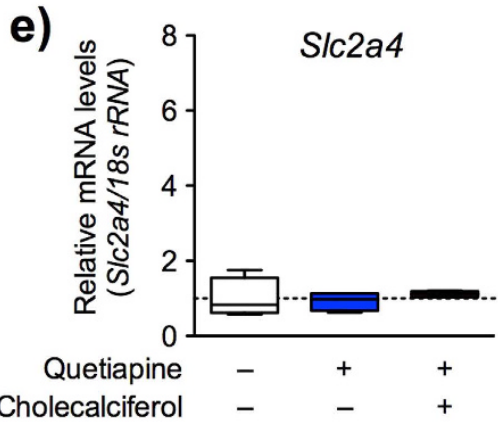

Figure 3. Effects of quetiapine and vitamin D/cholecalciferol supplementation on the expression levels of genes related to the insulin receptor signalling pathway. Mice were fed a control or cholecalciferolsupplemented diet and given quetiapine $(10 \mathrm{mg} / \mathrm{kg}$, i.p.) or vehicle, as described in Fig. 1. Immediately after a 150 min glucose tolerance test, the right thigh muscles were collected, and the gene expression levels of $\operatorname{Ins} r(\mathbf{a})$, Irs1 (b), Pik3r1 (c), Akt2 (d) and Slc2a4 (e) were analysed by quantitative real-time RT-PCR. Each expression level was normalised to that of $18 s \mathrm{r} R A$ and presented relative to the group receiving the control diet plus vehicle. Data are shown as box-whisker plots, where the horizontal lines indicate the medians, the boxes indicate the 25 th to 75 th percentiles and the whiskers indicate the entire range $\left(n=4-8 ;{ }^{*} p<0.05\right)$.

$$
\mathrm{OR}=\frac{a / b}{c / d}, 95 \% \mathrm{CI}=\exp \left(\log (\mathrm{OR}) \pm 1.96 \sqrt{\frac{1}{a}+\frac{1}{b}+\frac{1}{c}+\frac{1}{d}}\right)
$$

where $a, b, c$ and $d$ refer to the number of individuals in each group, and log refers to the natural logarithm. If the lower limit of the $95 \%$ CI was $>1$, a significant association was assumed between use of the drug of interest (i.e., quetiapine) and the increased occurrence of DM-related adverse events. Contrarily, if the upper limit of the $95 \%$ CI was $<1$, a significant association was assumed between use of the drug of interest (i.e., vitamin D) and the decreased occurrence of DM-related adverse events.

The search terms for "DM" and "vitamin D" are described in Supplementary Tables 1 and 2, respectively. We regarded drug indication data as the patients' primary diseases. To search for concomitant drugs associated with a decreased occurrence of quetiapine-induced, DM-related adverse events, the number of individuals treated with the concomitant drug of interest was restricted to a minimum of 1000, because ORs are prone to fluctuation when the sample size is small.

Combinatorial analysis of the GEO and KEGG PATHWAY databases. Microarray gene expression profiles were obtained from a previous study published in the GEO database (GEO Accession No. GSE 59923; http://www.ncbi.nlm.nih.gov/geo/query/acc.cgi? acc=GSE59923). Expression data were analysed in the livers of rats orally treated with quetiapine $(500 \mathrm{mg} / \mathrm{kg}, 5$ days of exposure with daily dosing) or vehicle (carboxymethylcellulose). The analysis included genes participating in the insulin resistance pathway in skeletal muscle cells, and obtained from the KEGG PATHWAY database (Entry No. map04931; http://www.kegg.jp/dbget-bin/ www_bget? pathway + map04931). For the expression analysis of each gene, log2-transformed microarray data were back-transformed to the original scale and presented relative to the vehicle-treated group.

Animals. All animal experiments were approved by the Kyoto University Animal Research Committee in accordance with the ethical guidelines of the Committee. All experiments were designed to minimise the use of animals and number of experiments. Male ICR mice (5-6 weeks of age) were purchased from Japan SLC (Shizuoka, Japan) and housed with a constant ambient temperature $\left(24 \pm 1{ }^{\circ} \mathrm{C}\right)$ and humidity $(55 \% \pm 10 \%)$ on a $12 \mathrm{~h} / 12 \mathrm{~h} \mathrm{light/dark}$ cycle. Food and water were freely available, except for during the fasting period before the glucose tolerance test. 
a)

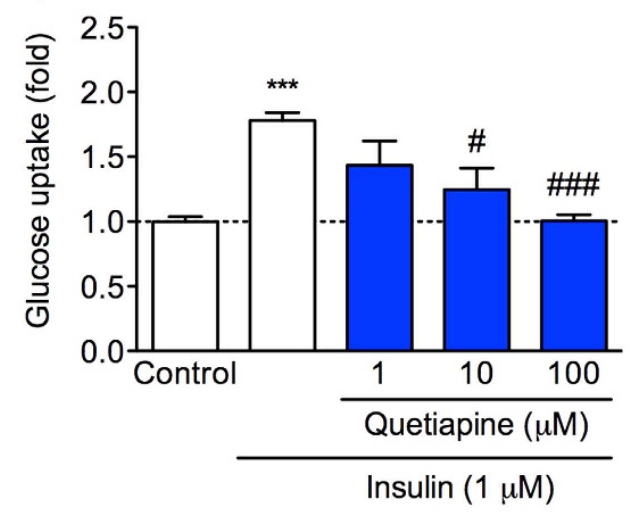

c)

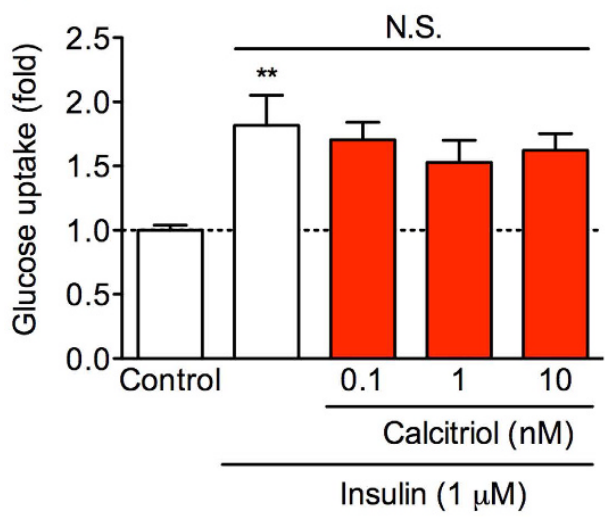

b)
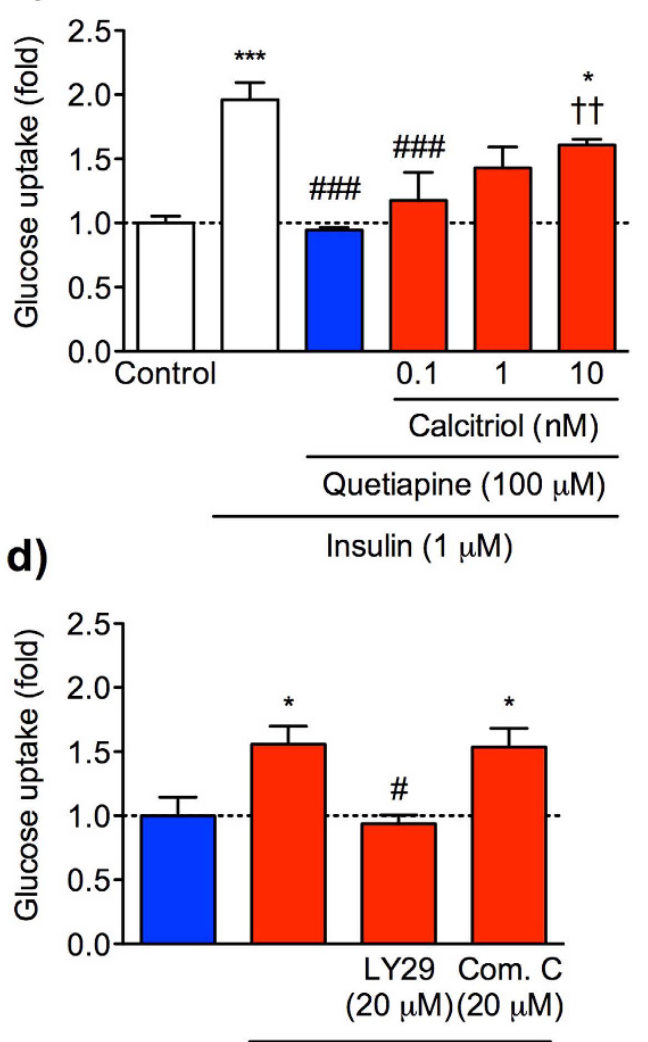

$$
\begin{aligned}
& \text { Calcitriol (10 nM) } \\
& \text { Insulin }(1 \mu \mathrm{M})+\text { Quetiapine }(100 \mu \mathrm{M})
\end{aligned}
$$

Figure 4. Calcitriol improved quetiapine-induced insulin resistance through PI3K signalling pathway in C2C12 myotubes. Glucose uptake was evaluated using 2-NBDG as described in Materials and Methods. (a) Concentration-dependent effect of quetiapine on insulin-stimulated glucose uptake. Differentiated C2C12 cells were treated with quetiapine $(1-100 \mu \mathrm{M})$ for $1 \mathrm{~h}$. The cells were then stimulated with or without insulin $(1 \mu \mathrm{M})$ for $15 \mathrm{~min} . n=10 .{ }^{* *} p<0.001$ vs. control group; ${ }^{\#} p<0.05,{ }^{\# \#} p<0.001$ vs. insulin-treated group. (b) Concentration-dependent effect of calcitriol on quetiapine-induced insulin resistance. Differentiated $\mathrm{C} 2 \mathrm{C} 12$ cells were treated with calcitriol $(0.1-10 \mathrm{nM})$ for $24 \mathrm{~h}$, followed by quetiapine $(100 \mu \mathrm{M})$ for $1 \mathrm{~h}$. The cells were then stimulated with or without insulin $(1 \mu \mathrm{M})$ for $15 \mathrm{~min} . n=10$. ${ }^{*} p<0.05,{ }^{* * *} p<0.001$ vs. control group; ${ }^{\# \# \#} p<0.001$ vs. insulin-treated group; ${ }^{\dagger \dagger} p<0.01$ vs. quetiapine-treated group. (c) Null effect of calcitriol on insulin-stimulated glucose uptake. Differentiated C2C12 cells were treated with calcitriol $(0.1-10 \mathrm{nM})$ for $24 \mathrm{~h}$. The cells were then stimulated with or without insulin $(1 \mu \mathrm{M})$ for $15 \mathrm{~min} . n=9$. ${ }^{* *} p<0.01$ vs. control group; N.S. = not significant. (d) Effect of LY294002 (PI3K inhibitor) or compound C (AMPK inhibitor) on the improvement of quetiapine-induced insulin resistance by calcitriol. Differentiated C2C12 cells were treated with calcitriol $(10 \mathrm{nM})$ for $24 \mathrm{~h}$, followed by quetiapine $(100 \mu \mathrm{M})$ in the presence or absence of LY294002 (LY29; $20 \mu \mathrm{M})$ or compound C (Com. C; $20 \mu \mathrm{M})$ for $1 \mathrm{~h}$. The cells were then stimulated with insulin $(1 \mu \mathrm{M})$ for $15 \mathrm{~min}$. $n=9-13,{ }^{\star} p<0.05$ vs. quetiapine-treated group; ${ }^{*} p<0.05$ vs. quetiapine and calcitriol-treated group. Data are given as means \pm SEM relative to the control $(\mathbf{a}-\mathbf{c})$ or insulin and quetiapine-treated (d) group.

Drugs and reagents. Quetiapine was purchased from Wako Pure Chemical Industries (Osaka, Japan). Methamphetamine was from Sumitomo Dainippon Pharma (Osaka, Japan). D-Glucose was from Nacalai Tesque (Kyoto, Japan). Insulin was from Biological Industries (Cromwell, CT, USA). 2-Deoxy-2-[(7-nitro2,1,3-benzoxadiazol-4-yl)amino]-D-glucose (2-NBDG), LY294002, and dorsomorphin (compound C) were from Cayman Chemical (Ann Arbor, MI, USA). Calcitriol was from Toronto Research Chemicals (Ontario, Canada). Dulbecco's modified Eagle's medium (DMEM) and foetal bovine serum (FBS) were from Sigma-Aldrich (Saint-Louis, MO, USA). Horse serum (HS) was from Invitrogen Japan (Tokyo, Japan).

For in vivo study, quetiapine and methamphetamine were dissolved in distilled water plus $1 \%$ Tween 80 prior to use. D-Glucose was dissolved in distilled water before use. Quetiapine, methamphetamine and D-glucose were administered intraperitoneally at a volume of $10 \mathrm{ml} / \mathrm{kg}$ body weight.

Vitamin $\mathrm{D}_{3}$ (cholecalciferol) was administered orally in the diet. MF diet (Oriental Yeast, Tokyo, Japan) containing $1370 \mathrm{IU}$ vitamin $\mathrm{D}_{3} / \mathrm{kg}$ ( $8 \mathrm{IU}$ vitamin $\mathrm{D}_{3} /$ day based on daily consumption of $6 \mathrm{~g}$ chow $/ 30 \mathrm{~g}$ body weight) served as the control diet. The cholecalciferol-supplemented diet consisted of a mixture of MF diet and 200,000 $\mathrm{IU}$ vitamin $\mathrm{D}_{3} / \mathrm{kg}$ (1200 IU/day). 
Intraperitoneal glucose tolerance test. Mice were fed a control or cholecalciferol-supplemented diet for 1 week, followed by a $16 \mathrm{~h}$ fast before a $150 \mathrm{~min}$ intraperitoneal glucose tolerance test. Quetiapine $(10 \mathrm{mg} / \mathrm{kg}) \mathrm{or}$ vehicle ( $1 \%$ Tween 80$)$ was administered intraperitoneally before the injection of D-glucose ( $3 \mathrm{~g} / \mathrm{kg})$. Blood samples were collected from the tail vein at the indicated time points, and blood glucose levels were measured using an Accu-Chek Blood Glucose Meter (Roche Diagnostics, Almere, the Netherlands). Blood insulin levels were measured using a Mouse Insulin Enzyme-Linked Immunosorbent Assay Kit (Morinaga Institute of Biological Science, Yokohama, Japan) and $5 \mu \mathrm{l}$ blood samples collected at the indicated time points, as instructed by the manufacturer.

Real-time quantitative RT-PCR. Immediately after the glucose tolerance test, mice were sacrificed by cervical dislocation, and the right thigh muscles were collected. Total RNA was isolated using the ISOGEN Reagent (Nippon Gene, Tokyo, Japan), and the isolated RNA $(0.5 \mu \mathrm{g})$ was reverse transcribed using the ReverTra Ace qPCR RT Kit (Toyobo, Osaka, Japan). Real-time quantitative RT-PCR was performed using the StepOne Real-Time PCR System (Life Technologies, Carlsbad, CA, USA) and the THUNDERBIRD SYBR qPCR Mix (Toyobo). Each PCR amplification consisted of heat activation for $10 \mathrm{~min}$ at $95^{\circ} \mathrm{C}$, followed by 40 cycles at $95^{\circ} \mathrm{C}$ for $15 \mathrm{~s}$ and $60^{\circ} \mathrm{C}$ for $1 \mathrm{~min}$.

The oligonucleotide primers used for RT-PCR were as follows: $5^{\prime}$-GTA ACC CGT TGA ACC CCA TT- $3^{\prime}$ and $5^{\prime}$-CCA TCC AAT CGG TAG TAG CG-3' for the $18 \mathrm{~s}$ ribosomal RNA gene (18s $\left.r R N A\right)$; $5^{\prime}$-CCC AGG CCA TCC CGA AAG- $3^{\prime}$ and 5' ${ }^{\prime}$-TCT CAA ATG GCC TGT GCT CC-3' for Insr; $5^{\prime}$-TTA GGC AGC AAT GAG GGC AA-3' and $5^{\prime}$-TCT TCA TTC TGC TGT GAT GTC CA-3' for Irs 1 ; $5^{\prime}$-GAC AGC GAA GCG ACG GC-3' and $5^{\prime}-$ GTC TGA TTT TAC TGC CAC GCT C-3' for Pik3r1; $5^{\prime}$-CGC TTG CGG TCT GAT GTT TT- $3^{\prime}$ and $5^{\prime}$-AAT ACC GCC TTT TCC AGC CA- $3^{\prime}$ for $A k t 2$; and $5^{\prime}$-TTA TTG CAG CGC CTG AGT CT- $3^{\prime}$ and $5^{\prime}$-GGG TTC CCC ATC GTC AGA G-3' for Slc2a4. The mRNA expression levels of each gene were normalised to that of $18 s$ s $R A$, which was measured in parallel in each sample and expressed relative to the vehicle-treated group.

Cell culture and differentiation. Mouse C2C12 myoblast cell line was kindly provided by Prof. H. Takeshima (Kyoto University, Graduate School of Pharmaceutical Sciences, Kyoto, Japan). The cells were cultured in 100-mm dishes in DMEM containing $10 \%$ heat-inactivated $\mathrm{FBS}$ at $37^{\circ} \mathrm{C}$ with $5 \% \mathrm{CO}_{2}$. One day after seeding cells in black 96 -well plates ( $>70 \%$ confluence), the medium was switched to DMEM with $2 \%$ HS to differentiate cells into myotubes. The myotubes were used for experiments 3-5 days following differentiation.

Glucose uptake assay. Glucose uptake assay was performed by measuring the uptake of 2-NBDG, a fluorescent derivative of glucose. Differentiated myotubes were starved in a serum-free DMEM for $3 \mathrm{~h}$ before treatment with the drugs. After treatment, cells were stimulated with or without insulin $(1 \mu \mathrm{M})$ dissolved in $\mathrm{KRPH}$ buffer (136 mM NaCl, $4.7 \mathrm{mM} \mathrm{KCl}, 1 \mathrm{mM} \mathrm{MgSO}_{4}, 1 \mathrm{mM} \mathrm{CaCl}_{2}, 5 \mathrm{mM} \mathrm{KH}_{2} \mathrm{PO}_{4}, 20 \mathrm{mM}_{\mathrm{HEPES}}$ ) for $15 \mathrm{~min}$ followed by the addition of 2-NBDG $(50 \mu \mathrm{M})$ for $20 \mathrm{~min}$. After incubation, free 2-NBDG was washed out 3 times with KRPH buffer. The fluorescence retained in the cells was measured with a fluorescence microplate reader (FDSS/ $\mu$ Cell; Hamamatsu Photonics, Shizuoka, Japan) at an excitation wavelength of $480 \mathrm{~nm}$ and an emission wavelength of $540 \mathrm{~nm}$.

Statistical analysis. Statistical analyses of the FAERS and GEO databases were performed with $\mathrm{R}$ version 3.1.2 Software (R Foundation for Statistical Computing, Vienna, Austria). Data obtained from the animal experiments were analysed with GraphPad Prism 5 Software (GraphPad, San Diego, CA, USA). Differences between two groups were compared via an unpaired two-tailed $t$-test with Welch's correction, while differences between more than two groups were compared via the Kruskal-Wallis test followed by Dunn's post hoc test (Fig. 3) or the one-way ANOVA followed by Tukey's post hoc test (Fig. 4). Time-course data were analysed by applying a two-way ANOVA for repeated measures, followed by the Bonferroni post hoc test. Differences were considered significant at $p<0.05$.

\section{References}

1. American Diabetes Association, American Psychiatric Association, American Association of Clinical Endocrinologists \& North American Association for the Study of Obesity. Consensus development conference on antipsychotic drugs and obesity and diabetes. Diabetes Care 2, 596-601 (2004).

2. Sernyak, M. J. et al. Association of diabetes mellitus with use of atypical neuroleptics in the treatment of schizophrenia. Am. J. Psychiatry 159, 561-566 (2002).

3. Ely, S. F., Neitzel, A. R. \& Gill, J. R. Fatal diabetic ketoacidosis and antipsychotic medication. J. Forensic Sci. 58, 398-403 (2013).

4. Buchholz, S., Morrow, A. F. \& Coleman, P. L. Atypical antipsychotic-induced diabetes mellitus: an update on epidemiology and postulated mechanisms. Intern. Med. J. 38, 602-606 (2008).

5. Rojo, L. E. et al. Metabolic syndrome and obesity among users of second generation antipsychotics: A global challenge for modern psychopharmacology. Pharmacol. Res. 101, 74-85 (2015).

6. Tatonetti, N. P. et al. Detecting drug interactions from adverse-event reports: interaction between paroxetine and pravastatin increases blood glucose levels. Clin. Pharmacol. Ther. 90, 133-142 (2011).

7. Tatonetti, N. P., Ye, P. P., Daneshjou, R. \& Altman, R. B. Data-driven prediction of drug effects and interactions. Sci. Transl. Med. 4, $125 \mathrm{ra3} 1$ (2012).

8. Zhao, S. et al. Systems pharmacology of adverse event mitigation by drug combinations. Sci. Transl. Med. 5, 206ra140 (2013).

9. Kanehisa, M. \& Goto, S. KEGG: Kyoto encyclopedia of genes and genomes. Nucleic Acids Res. 28, 27-30 (2000).

10. Kanehisa, M. et al. KEGG as a reference resource for gene and protein annotation. Nucleic Acids Res. 44 (Database issue), D457-462 (2016).

11. Ganter, B., Snyder, R. D., Halbert, D. N. \& Lee, M. D. Toxicogenomics in drug discovery and development: mechanistic analysis of compound/class-dependent effects using the DrugMatrix database. Pharmacogenomics 7, 1025-1044 (2006). 
12. Edgar, R., Domrachev, M. \& Lash, A. E. Gene Expression Omnibus: NCBI gene expression and hybridization array data repository. Nucleic Acids Res. 30, 207-210 (2002).

13. Barrett, T. et al. NCBI GEO: archive for functional genomics data sets-update. Nucleic Acids Res. 41 (Database issue), D991-995 (2013).

14. Reaven, G. M. Banting lecture 1988. Role of insulin resistance in human disease. Diabetes 37, 1595-1607 (1988).

15. Barroso, I. et al. Candidate gene association study in type 2 diabetes indicates a role for genes involved in beta-cell function as well as insulin action. PLoS Biol. 1, E20 (2003).

16. Thauvin-Robinet, C. et al. PIK3R1 mutations cause syndromic insulin resistance with lipoatrophy. Am. J. Hum. Genet. 93, 141-149 (2013).

17. DeFronzo, R. A. \& Tripathy, D. Skeletal muscle insulin resistance is the primary defect in type 2 diabetes. Diabetes Care 32, S157-193 (2009).

18. Maestro, B., Dávila, N., Carranza, M. C. \& Calle, C. Identification of a Vitamin D response element in the human insulin receptor gene promoter. J. Steroid Biochem. Mol. Biol. 84, 223-230 (2003).

19. Nwosu, B. U. et al. A potential role for adjunctive vitamin D therapy in the management of weight gain and metabolic side effects of second-generation antipsychotics. J. Pediatr. Endocrinol. Metab. 24, 619-626 (2011).

20. Thakurathi, N. et al. Open-label pilot study on vitamin $\mathrm{D}_{3}$ supplementation for antipsychotic-associated metabolic anomalies. Int. Clin. Psychopharmacol. 28, 275-282 (2013).

21. Dang, R. et al. Vitamin D deficiency exacerbates atypical antipsychotic-induced metabolic side effects in rats: involvement of the INSIG/SREBP pathway. Eur. Neuropsychopharmacol. 25, 1239-1247 (2015).

22. Pittas, A. G., Lau, J., Hu, F. B. \& Dawson-Hughes, B. The role of vitamin D and calcium in type 2 diabetes. A systematic review and meta-analysis. J. Clin. Endocrinol. Metab. 92, 2017-2029 (2007).

23. Miller, D. D. Review and management of clozapine side effects. J. Clin. Psychiatry 61, 14-17 (2000),

24. Haussler, M. R. et al. Molecular mechanisms of vitamin D action. Calcif. Tissue Int. 92, 77-98 (2013).

25. Luo, J. et al. Loss of class IA PI3K signaling in muscle leads to impaired muscle growth, insulin response, and hyperlipidemia. Cell Metab. 3, 355-366 (2006).

26. Thong, F. S., Dugani, C. B. \& Klip, A. Turning signals on and off: GLUT4 traffic in the insulin-signaling highway. Physiology (Bethesda) 20, 271-284 (2005).

27. Engl, J. et al. Olanzapine impairs glycogen synthesis and insulin signaling in L6 skeletal muscle cells. Mol. Psychiatry 10, 1089-96 (2005).

28. Panariello, F. et al. Clozapine impairs insulin action by up-regulating Akt phosphorylation and Ped/Pea-15 protein abundance. J. Cell. Physiol. 227, 1485-1492 (2012).

29. Zhou, Q. G. et al. 1,25-Dihydroxyvitamin D improved the free fatty-acid-induced insulin resistance in cultured C2C12 cells. Diabetes Metab. Res. Rev. 24, 459-464 (2008).

30. Ngai, Y. F. et al. Quetiapine treatment in youth is associated with decreased insulin secretion. J. Clin. Psychopharmacol. 34, 359-364 (2014).

31. Ozasa, R. et al. The antipsychotic olanzapine induces apoptosis in insulin-secreting pancreatic $\beta$ cells by blocking PERK-mediated translational attenuation. Cell Struct. Funct. 38, 183-195 (2013).

32. Lorberbaum, T. et al. Systems pharmacology augments drug safety surveillance. Clin. Pharmacol. Ther. 97, 151-158 (2015).

33. Kim, H. et al. Meta-analysis of large-scale toxicogenomic data finds neuronal regeneration related protein and cathepsin D to be novel biomarkers of drug-induced toxicity. PLoS One 10, e0136698 (2015).

34. Sakaeda, T., Tamon, A., Kadoyama, K. \& Okuno, Y. Data mining of the public version of the FDA adverse event reporting system. Int. J. Med. Sci. 10, 796-803 (2013).

\section{Acknowledgements}

This work was supported in part by Grants-in-Aid for Scientific Research (KAKENHI) from the Japanese Society for the Promotion of Science.

\section{Author Contributions}

T. Nagashima and S.K. designed the experiments; T. Nagashima analysed FAERS, GEO and KEGG PATHWAY databases, and performed the experiments; T. Nagashima, H.S., T. Nakagawa and S.K. analysed the data; T. Nagashima, T. Nakagawa and S.K. wrote the manuscript.

\section{Additional Information}

Supplementary information accompanies this paper at http://www.nature.com/srep

Competing financial interests: The authors declare no competing financial interests.

How to cite this article: Nagashima, T. et al. Prevention of antipsychotic-induced hyperglycaemia by vitamin $\mathrm{D}$ : a data mining prediction followed by experimental exploration of the molecular mechanism. Sci. Rep. 6, 26375; doi: 10.1038/srep26375 (2016).

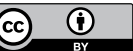

This work is licensed under a Creative Commons Attribution 4.0 International License. The images or other third party material in this article are included in the article's Creative Commons license, unless indicated otherwise in the credit line; if the material is not included under the Creative Commons license, users will need to obtain permission from the license holder to reproduce the material. To view a copy of this license, visit http://creativecommons.org/licenses/by/4.0/ 